\title{
The Effect of Channel State Information on Optimum Energy Allocation and Energy Efficiency of Cooperative Wireless Transmission Systems
}

\author{
Jie Yang and D. Richard Brown III \\ \{abbyyang,drb\}@wpi.edu
}

\begin{abstract}
This paper considers the problem of how to efficiently allocate transmission energy in a wireless communication system with two delay-constrained cooperating sources and one destination. The sources in the system relay each other's transmissions via an orthogonal amplify-and-forward protocol. The channels are assumed to be flat fading and the sources are each required to satisfy an outage probability constraint. The analysis focuses on optimum energy allocation and energy efficiency for two distinctly different scenarios: (i) the sources have access to partial channel state information (the instantaneous channel amplitudes) and (ii) the sources have access to only the channel statistics. Numerical examples are presented for independent Rayleigh fading channels demonstrating that partial channel state information significantly improves the energy efficiency of cooperative transmission. Our results also show that, while cooperative transmission tends to have better energy efficiency than direct (noncooperative) transmission, opportunistic direct transmission with partial channel state information is often more energy efficient than cooperative transmission without knowledge of the channel state.
\end{abstract}

\section{INTRODUCTION}

Spatial diversity can be achieved in multiuser communication systems through user cooperation where transmitters and/or receivers pool their antenna resources to form a "virtual antenna array". Transmit cooperation was first proposed in [1] and has since been shown to offer many of the benefits of multi-antenna transmission, e.g. increased rate and/or reduced outage probability, to transmitters with single antennas [2]-[6].

Unlike a single transmitter employing a dedicated antenna array, cooperating transmitters each have their own local resources. It was first suggested in [1] that cooperation could lead to an overall reduction in transmit energy and, consequently, increased battery life for batterypowered transmitters. The problem of optimum energy allocation in cooperative networks is not straightforward, however, and depends to a large extent on the choice of cooperative protocol and performance measure. Energy allocation for decode-and-forward cooperation [7] was analyzed with the goal of maximizing rate in [8], minimizing outage probability in [9], and satisfying a fixed outage

Authors are with the Electrical and Computer Engineering Department, Worcester Polytechnic Institute, Worcester, MA. This work was supported by NSF award CCF-0447743. probability target in [10]. Energy allocation for amplifyand-forward cooperation was analyzed with the goal of minimizing BER in [11], minimizing total power subject to a rate constraint in [12], and minimizing total power subject to fixed SNR and outage probability constraints in [13] and [14], respectively. Minimum outage probability energy allocation has also been considered for a hybrid protocol in [15].

While [9] considered the impact of partial channel state information (specifically, the instantaneous channel amplitudes) at the transmitters on the outage probability performance of the decode-and-forward protocol, the impact of channel state information on amplify-and-forward cooperative transmission has not been investigated. This paper considers the problem of optimum energy allocation and energy efficiency of the two-source amplify-and-forward protocol in two different scenarios: (i) the sources have access to the partial channel state information (PCSIT) and (ii) the sources have access to only the channel statistics. In both cases, the sources are assumed to be delay-constrained and are required to satisfy an outage probability constraint. We derive the optimum (minimum energy) opportunistic energy allocation strategy for the case with PCSIT and explicitly describe the set of channel conditions under which cooperation is not energy efficient. For the case without CSIT, we analyze the outage probability and derive explicit bounds for the case with independent Rayleigh fading channels. These bounds are then used to derive the optimum fixed energy allocation strategy for the case when the sources do not have CSIT.

Numerical examples are presented for independent Rayleigh fading channels demonstrating that PCSIT significantly improves the energy efficiency of cooperative transmission. Our results also show that, while cooperative transmission tends to have better energy efficiency than direct (noncooperative) transmission, opportunistic direct transmission with PCSIT is often more energy efficient than cooperative transmission without knowledge of the channel state.

\section{System Model and Cooperative Protocol}

We consider the two-source one-destination cooperative transmission system model shown in Figure 1. Each source is assumed to transmit in an orthogonal subchannel (e.g. FDMA). Both sources wish to communicate distinct 
information to the destination and cooperate via the "amplify-and-forward" protocol first described in [16]. The two-source amplify-and-forward cooperative transmission protocol divides the transmission interval into two timeslots of equal duration. Each source transmits its own information in the first timeslot (while receiving the transmission of the other source) and the second timeslot is used for cooperative retransmission of the signal received during the first timeslot. The channels are assumed to be flat and block-fading where their value is randomly generated but remains constant over the both timeslots in the cooperative frame. Note that each source transmits while receiving the transmission of the other source in the first timeslot. The sources operate in half-duplex mode, however, in the sense that transmission and reception does not occur simultaneously in any orthogonal subchannel.

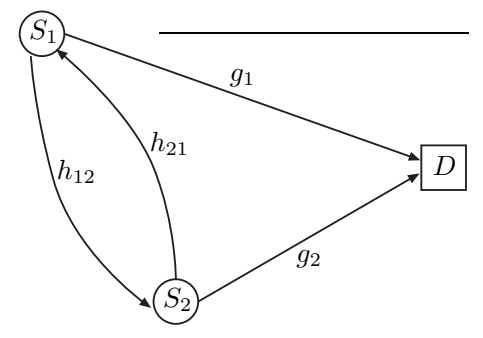

Fig. 1. Two-source one-destination cooperative transmission system model.

In the two-source amplify-and-forward scenario, there are a total of four transmission energies in each cooperative frame. We denote these as $\mathcal{E}_{i}(k)$ where $i \in\{1,2\}$ and $k \in$ $\{1,2\}$ denote the source and timeslot indices, respectively. Note that, since the sources are restricted to cooperative retransmission in the second timeslot, the SNR of the $i^{\text {th }}$ source is affected only by $\mathcal{E}_{i}(1)$ and $\mathcal{E}_{j}(2)$ for $j \neq i$.

To ease notation in the following sections, we consider, due to the symmetry of the problem, the $i^{\text {th }}$ source as the "source" and the $j^{\text {th }}$ source as the "relay". We denote $\mathcal{E}_{s}:=\mathcal{E}_{i}(1)$ and $\mathcal{E}_{r}:=\mathcal{E}_{j}(2)$ as the transmission energies relevant to the SNR of the $i^{\text {th }}$ source and $\mathcal{E}:=\mathcal{E}_{s}+\mathcal{E}_{r}$ as the total transmission energy used in the transmission of the information from the $i^{\text {th }}$ source to the destination. We also denote $G_{s}:=g_{i}^{2} / \sigma^{2}, G_{r}:=g_{j}^{2} / \sigma^{2}$, and $H:=h_{i j}^{2} / \sigma^{2}$ as the normalized squared channel gains in the cooperative communication system shown in Figure 1 where $\sigma^{2}>0$ denotes the variance of the zero-mean Gaussian noise present in the channel.

\section{Destination Processing And SNR Analysis}

The performance measure that we consider in this paper is outage probability, defined as the probability that the SNR of the source's information at the destination falls below a deterministic threshold $\rho$, i.e.,

$$
p:=\operatorname{Prob}[\text { outage }]=\operatorname{Prob}[\operatorname{SNR}<\rho] .
$$

The SNR of the sources's information at the destination is determined not only by the channel states and the transmission energies but also by how the destination forms its decision statistic from the received source and relay transmissions. To better isolate the effect of channel state information at the source and relay, we assume that the destination has full access to the channel states and transmit energies of both sources in both timeslots and uses maximal ratio combining (MRC) of the relevant source/relay observations in both timeslots to maximize the SNR of the decision statistic.

When the source and relay have access to PCSIT, they can dynamically allocate their transmission energies according to the instantaneous channel amplitudes in each transmission interval. The resulting instantaneous SNR at the destination, after MRC, can be expressed as

$$
\mathrm{SNR}_{\text {pcsit }}=G_{s} \mathcal{E}_{s}+\frac{H \mathcal{E}_{s} G_{r} \mathcal{E}_{r}}{1+H \mathcal{E}_{s}+G_{r} \mathcal{E}_{r}} .
$$

When the source and relay do not have access to the channel state, they cannot dynamically allocate their transmission energies in each transmission interval. Instead, they must select a fixed transmission energy based only on knowledge of the channel statistics. The resulting instantaneous SNR at the destination, after MRC, can be expressed as

$$
\mathrm{SNR}_{\text {nocsit }}=G_{s} \mathcal{E}_{s}+\frac{H \mathcal{E}_{s} G_{r} \mathcal{E}_{r}}{1+\mathrm{E}[H] \mathcal{E}_{s}+G_{r} \mathcal{E}_{r}} .
$$

It may be somewhat surprising that (1) and (2) appear to be almost identical. In both cases, the instantaneous SNR at the destination is fully specified by the normalized channel amplitudes and transmit energies. The fundamental difference between (1) and (2), however, is in how the transmit energies $\mathcal{E}_{s}$ and $\mathcal{E}_{r}$ are selected. In (1), the transmit energies are functions of the current channel states $G_{s}$, $G_{r}$, and $H$ whereas, in (2), these energies are based only on knowledge of the channel statistics, e.g., $\mathrm{E}\left[G_{s}\right], \mathrm{E}\left[G_{r}\right]$, and $\mathrm{E}[H]$. The following sections analyze the significance of this difference in terms of optimum energy allocation strategies and the energy efficiency of the two-source cooperative transmission system.

\section{Optimum Energy Allocation with PCSIT}

To facilitate energy allocation analysis for general $p>0$, we first consider the case when $p=0$. The problem in this case is to select an energy allocation $\left\{\mathcal{E}_{s}, \mathcal{E}_{r}\right\}$ such that $\mathrm{SNR}_{\text {pcsit }} \geq \rho$ almost surely. Since the source and relay have access to the instantaneous channel amplitudes, they can dynamically allocate their transmission energies such that the randomness induced by the channel state in $S N R_{\text {pcsit }}$ is removed and $S_{N R} R_{\text {pcsit }}=\rho$. There are, however, an infinite number of energy allocations that satisfy $\operatorname{SNR}_{\mathrm{pcsit}}=\rho$. The space of admissible energy allocations satisfying $\mathrm{SNR}_{\mathrm{pcsit}}=$ $\rho$ can be described as the region in $\mathbb{R}^{2}$ where $\mathcal{E}_{r} \geq 0$ and $\frac{\rho}{H+G_{s}}<\mathcal{E}_{s} \leq \frac{\rho}{G_{s}}$, where the upper limit to $\mathcal{E}_{s}$ corresponds to the case when $\mathcal{E}_{r}=0$ (direct transmission or, equivalently, no cooperation) and the lower limit corresponds to the case when $\mathcal{E}_{r} \rightarrow \infty$ (infinite cooperaton). In the case 
of direct transmission, the total energy required to meet the SNR target is $\mathcal{E}=\mathcal{E}_{s}=\frac{\rho}{G_{s}}$.

Before deriving the optimum (minimum total energy $\left.\mathcal{E}_{s}+\mathcal{E}_{r}\right)$ cooperative energy allocation strategy in this scenario, we first consider the question of when is it more efficient for the relay to not transmit. This is made formal in the following proposition.

Proposition 1: There exists $\mathcal{E}<\frac{\rho}{G_{s}}$ if and only if

$$
\frac{G_{r}}{G_{s}}>1+\frac{G_{s}}{H \rho} \text {. }
$$

Proof: Using (1), the total energy required to satisfy the constraint $\mathrm{SNR}_{\text {pcsit }}=\rho$ can be written as

$$
\mathcal{E}:=\mathcal{E}_{s}+\mathcal{E}_{r}=\mathcal{E}_{s}+\frac{H \mathcal{E}_{s}^{2} G_{s}+\left(G_{s}-H \rho\right) \mathcal{E}_{s}-\rho}{G_{r}\left(\rho-\left(H+G_{s}\right) \mathcal{E}_{s}\right)} .
$$

Define the interval $\mathcal{A}=\left(\frac{\rho}{H+G_{s}}, \frac{\rho}{G_{s}}\right]$. If

$$
\arg \min _{\mathcal{E}_{s} \in \mathcal{A}} \mathcal{E}=\frac{\rho}{G_{s}}
$$

then $\mathcal{E}_{r}=0$ and $\mathcal{E}$ is minimized with direct transmission. Otherwise, $\mathcal{E}_{r}>0$ and cooperative transmission minimizes $\mathcal{E}$.

In order to determine if the minimum of (4) on $\mathcal{A}$ occurs at the point $\mathcal{E}_{s}=\frac{\rho}{G_{s}}$, we first establish that (4) can have only one minimum on $\mathcal{A}$ by proving that (4) is a strictly convex function of $\mathcal{E}_{s}$ on $\mathcal{A}$. The second derivative of (4) with respect to $\mathcal{E}_{s}$ can be written as

$$
\mathcal{E}^{\prime \prime}:=\frac{\partial^{2}}{\partial \mathcal{E}_{s}^{2}} \mathcal{E}=\frac{-2 G_{r}^{2} H \rho\left[(\rho+1) H+G_{s}\right]}{G_{r}^{3}\left(\rho-\left(H+G_{s}\right) \mathcal{E}_{s}\right)^{3}} .
$$

Note that the numerator of (5) is a negative quantity not dependent on $\mathcal{E}_{s}$. Since $\mathcal{E}_{s}\left(H+G_{s}\right)>\rho$ and $G_{r}>0$, the denominator of (5) is also negative on the interval $\mathcal{E}_{s} \in \mathcal{A}$, hence $\mathcal{E}^{\prime \prime}$ is always positive on $\mathcal{A}$. This implies that $\mathcal{E}$ is a strictly convex function of $\mathcal{E}_{s}$ on $\mathcal{A}$.

Given the convexity of $\mathcal{E}$ on $\mathcal{A}$, we can determine whether the unique minimum of (4) on $\mathcal{A}$ occurs at the point $\mathcal{E}_{s}=\frac{\rho}{G_{s}}$ by evaluating the first derivative of (4) at this point. If the first derivative is positive, then the minimum of (4) on $\mathcal{A}$ must occur at $\mathcal{E}_{s}<\frac{\rho}{G_{s}}$ (corresponding to cooperative transmission), otherwise the minimum occurs at $\mathcal{E}_{s}=\frac{\rho}{G_{s}}$ (corresponding to direct transmission). The first derivative of (4) evaluated at $\mathcal{E}_{s}=\frac{\rho}{G_{s}}$ can be written as

$$
\mathcal{E}^{\prime}\left(\frac{\rho}{G_{s}}\right):=\frac{\partial}{\partial \mathcal{E}_{s}} \mathcal{E}\left(\frac{\rho}{G_{s}}\right)=1-\frac{G_{s}\left(H \rho+G_{s}\right)}{G_{r} H \rho}
$$

This quantity is positive if and only if the conditions of (3) are satisfied, hence the unique minimum of (4) on $\mathcal{A}$ must occur at $\mathcal{E}_{s}<\frac{\rho}{G_{s}}$ when the conditions of (3) are satisfied. Otherwise, the minimum of (4) on $\mathcal{A}$ must occur at $\mathcal{E}_{s}=\frac{\rho}{G_{s}}$ and direct transmission is optimum.

Proposition 1 implies that it is possible through cooperative transmission to achieve a reduction in the transmit energy required to meet a fixed SNR target only if the ratio of the relay-destination to source-destination channel gains exceeds some threshold greater than one. If this condition is not satisfied, it is more efficient for the source to satisfy its SNR target through direct transmission and for no energy to be expended by the relay in the cooperative timeslot. Moreover, since $G_{r} / G_{s}$ cannot be greater than one for both source 1 and source 2, Proposition 1 implies that at most only one source should cooperate in each transmission interval. In some cases, total energy is minimized if neither source cooperates and both sources satisfy their SNR targets via direct transmission.

When the conditions of Proposition 1 are satisfied, the energy allocation that minimizes $\mathcal{E}$ while satisfying $\mathrm{SNR}_{\text {pcsit }}=\rho$ can be determined through standard calculus techniques. The value of $\mathcal{E}_{s}$ that minimizes $\mathcal{E}$ subject to the fixed SNR constraint can be written as

$\mathcal{E}_{s}^{*}=\frac{\rho}{H+G_{s}}+\frac{(\rho H)^{1 / 2}\left(G_{s}+(1+\rho) H\right)^{1 / 2}}{\left(H+G_{s}\right)\left(H\left(G_{r}-G_{s}\right)+G_{s} G_{r}\right)^{1 / 2}}$

and $\mathcal{E}_{r}^{*}$ is implied through (6) and (1) given $\mathrm{SNR}_{\text {pcsit }}=\rho$. The minimum total transmission energy is then $\mathcal{E}^{*}=$ $\mathcal{E}_{s}^{*}+\mathcal{E}_{r}^{*}$. Note that the random nature of the channel state implies that $\mathcal{E}^{*}$ is random. We denote the cumulative distribution function of $\mathcal{E}^{*}$ satisfying $\mathrm{SNR}_{\text {pcsit }}=\rho$ as $F_{\mathcal{E}^{*}}(x)=\operatorname{Prob}\left[\mathcal{E}^{*} \leq x\right]$.

We now develop the optimum energy allocation strategy for the case when $p>0$. Let $t$ denote the value at which $F_{\mathcal{E}^{*}}(t)=1-p$. Given the current channel state, if $(3)$ is satisfied, solve for the optimum transmission energies $\mathcal{E}_{s}^{*}$ and $\mathcal{E}_{r}^{*}$ that satisfy $\mathrm{SNR}_{\mathrm{pcsit}}=\rho$ via $(6)$ and (1). If (3) is not satisfied, direct transmission is optimum and $\mathcal{E}_{s}^{*}=\frac{\rho}{G_{s}}$ and $\mathcal{E}_{r}^{*}=0$. Note that the resulting minimum total energy $\mathcal{E}^{*}=\mathcal{E}_{s}^{*}+\mathcal{E}_{r}^{*}$ will exceed the threshold $t$ with probability $p$. Since outage events are permitted with probability $p$, the strategy that minimizes average total transmission energy is to not transmit at all if $\mathcal{E}^{*}>t$. If $\mathcal{E}^{*} \leq t$, transmission occurs such that $\mathrm{SNR}_{\text {pcsit }}=\rho$ with the optimum energies $\mathcal{E}_{s}^{*}$ and $\mathcal{E}_{r}^{*}$. We note that this is essentially an opportunistic transmission strategy where the source and relay avoid transmission (and cause an outage) in cases when the channel state is unfavorable. The outage probability requirement is satisfied under this strategy since the SNR at the destination will be equal to $\rho$ with probability $1-p$ and equal to zero otherwise.

\section{Optimum Energy Allocation without CSIT}

When the source and relay do not have access to the channel state, they cannot dynamically allocate their transmit energy and must instead select fixed transmission energies based only on the channel statistics. We begin our analysis of optimum energy allocation in this scenario by first deriving expressions for the outage probability of the two-source cooperative transmission system assuming fixed transmission energies.

\section{A. Outage Probability Analysis and Bounds}

A general and exact expression for the outage probability in the fixed transmission energy scenario follows directly from the fact that the outage probability is the cumulative distribution function of the random variable 
SNR nocsit. Given the joint channel density $f_{G_{s}, G_{r}, H}(\boldsymbol{x})$, the outage probability can be expressed as

$$
p=\int_{R(\rho)} f_{G_{s}, G_{r}, H}(\boldsymbol{x}) d \boldsymbol{x}
$$

where the three-dimensional integration region $R(\rho)$ is derived from (2) and given as

$$
\begin{aligned}
R(\rho) & =\left\{\boldsymbol{x} \in \mathbb{R}^{3}: 0 \leq x_{1} \leq \frac{\rho}{\mathcal{E}_{s}}, 0 \leq x_{2}<\infty,\right. \\
& \text { and } \left.0 \leq x_{3} \leq \frac{\left(\rho-\mathcal{E}_{s} x_{1}\right)\left(1+\mathrm{E}[H] \mathcal{E}_{s}+\mathcal{E}_{r} x_{2}\right)}{\mathcal{E}_{s} \mathcal{E}_{r} x_{2}}\right\} .
\end{aligned}
$$

While the explicit description of the integration region $R(\rho)$ is straightforward, explicit analytical solutions to (7) are difficult to obtain in many common cases. To facilitate analysis, we present a pair of bounds to (7) below.

1) Lower Bound: Perfect Source-Relay Channel: A lower bound on the outage probability can be obtained by assuming that the channel between the sources $H$ is perfect, i.e. $H \rightarrow \infty$ and $\mathrm{E}[H] \rightarrow \infty$. In this case, (2) reduces to

$$
\mathrm{SNR}_{\text {nocsit }}=G_{s} \mathcal{E}_{s}+G_{r} \mathcal{E}_{r} .
$$

Evaluation of the outage probability in this case involves only two-dimensional integration of the joint channel density $f_{G_{s}, G_{r}}(\boldsymbol{x})$ over the region

$R_{l b}(\rho)=\left\{\boldsymbol{x} \in \mathbb{R}^{2}: 0 \leq x_{1} \leq \frac{\rho}{\mathcal{E}_{s}}\right.$ and $\left.0 \leq x_{2} \leq \frac{\rho-\mathcal{E}_{s} x_{1}}{\mathcal{E}_{r}}\right\}$.

In the case of independent Rayleigh fading channels, this bound can be evaluated explicitly. The random variables $G_{s}$ and $G_{r}$ are exponentially distributed in this case, with means denoted as $\mu_{s}$ and $\mu_{r}$, respectively. Evaluation of (7) with the integration region specified in (8) yields

$p \geq \frac{\mu_{s} \mathcal{E}_{s}\left(1-\exp \left(\frac{-\rho}{\mu_{s} \mathcal{E}_{s}}\right)\right)-\mu_{r} \mathcal{E}_{r}\left(1-\exp \left(\frac{-\rho}{\mu_{r} \mathcal{E}_{r}}\right)\right)}{\mu_{s} \mathcal{E}_{s}-\mu_{r} \mathcal{E}_{r}}$.

2) Upper Bound: Rectangular Integration Region: An upper bound on the outage probability expression (7) can be obtained by noting that slices of the (exact) threedimensional integration region $R(\rho)$ in the $x_{1}, x_{3}$ plane are triangular for all $x_{2}>0$. These triangular slices can be overbounded by enclosing rectangles with the identical intercepts to yield a slightly simplified integration region for $(7)$ as

$$
\begin{gathered}
R_{u b}(\rho)=\left\{\boldsymbol{x} \in \mathbb{R}^{3}: 0 \leq x_{1} \leq \frac{\rho}{\mathcal{E}_{s}}, 0 \leq x_{2}<\infty,\right. \\
\text { and } \left.0 \leq x_{3} \leq \frac{\rho\left(1+\mathrm{E}[H] \mathcal{E}_{s}+\mathcal{E}_{r} x_{2}\right)}{\mathcal{E}_{s} \mathcal{E}_{r} x_{2}}\right\} .
\end{gathered}
$$

where the key advantage with respect to the exact integration region is that $x_{1}$ has been eliminated from the upper limit of the integral over $x_{3}$. In the case of independent Rayleigh fading channels, this bound can be evaluated explicitly. Denote the means of the exponentially distributed random variables $G_{s}, G_{r}$, and $H$ as $\mu_{s}, \mu_{r}$, and
$\mu_{H}$, respectively. Evaluation of (7) with the integration region specified in (10) yields

$p \leq\left(1-\exp \left(\frac{-\rho}{\mu_{s} \mathcal{E}_{s}}\right)\right) \cdot\left(1-\exp \left(\frac{-\rho}{\mu_{H} \mathcal{E}_{s}}\right) \psi K_{1}(\psi)\right)$

where $K_{1}(\psi)$ is the modified Bessel function of the second kind and $\psi:=2 \sqrt{\frac{\rho\left(1+\mu_{H} \mathcal{E}_{s}\right)}{\mathcal{E}_{s} \mathcal{E}_{r} \mu_{r} \mu_{H}}}$. Note that the relay transmission energy $\mathcal{E}_{r}$ appears only in the denominator of $\psi$ in the upper bound (11).

\section{B. Optimum Energy Allocation}

While the outage probability bounds given in (9) and (11) are explicit and easily evaluated for any choice of transmit energies, analytical solutions to the optimum source/relay transmit energies based on these bounds are difficult to derive due to the fact that neither bound appears to yield an explicit solution for one transmit energy in terms of the other. Optimum energy allocations based on (9) and (11) can be obtained, however, using numerical optimization methods. The following section compares the numerical solutions to the optimum energy allocation and energy efficiency of cooperative transmission without CSIT to the analytical optimum energy allocation and energy efficiency of systems with PCSIT.

\section{Numerical EXAMPLES}

This section presents numerical examples demonstrating the impact of PCSIT on optimum cooperative energy allocation and energy efficiency for the case when the channels shown in Figure 1 are Rayleigh fading and independent. All of the results in this section assume $\mu_{H}=100$, and $\rho=10 \mathrm{~dB}$. Figures 2 and 5 consider the case when the relay has a statistically advantaged channel to the destination, i.e. $\mu_{r}=100$ and $\mu_{s}=10$. Figures 3 and 6 consider the case when the source and relay face statistically symmetric independent Rayleigh fading channels to the destination, i.e. $\mu_{r}=\mu_{s}=10$. Finally, Figures 4 and 7 consider the case when the relay has a statistically disadvantaged channel to the destination, i.e. $\mu_{r}=10$ and $\mu_{s}=100$.

Figures 2, 3, and 4 show the optimum source/relay energy allocations to achieve the outage probability target $p$ for the cases when the relay faces a statistically advantaged, symmetric, and disadvantaged channel to the destination, respectively. With PCSIT, the source always transmits with more energy than the relay and the energy gap grows as the relay's channel becomes less advantaged. This can be attributed the fact that the source must always transmit, but the relay will not cooperate with high probability when it faces a statistically disadvantaged channel to the destination, as implied in Proposition 1. Without CSIT, the optimum source and relay energies were numerically determined using the lower and upper bounds in (9) and (11), respectively. The upper bound results show that, in all cases without CSIT, the source transmits with more energy than the relay. In fact, the upper bound results suggest that the relay should not cooperate when its channel is symmetric or disadvantaged 


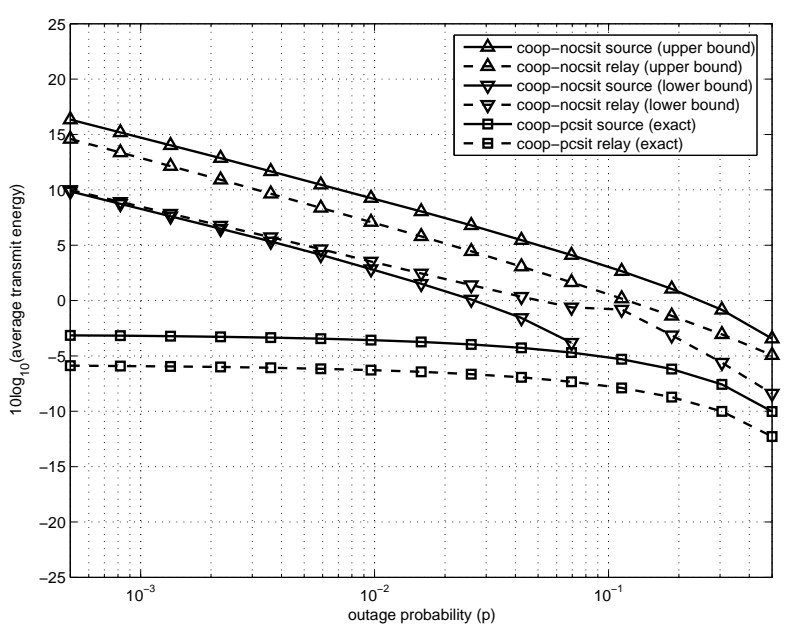

Fig. 2. Optimum energy allocation when the relay has a statistically advantaged channel to the destination. Note that the source does not transmit for $p>0.07$ when using the lower bound (9).

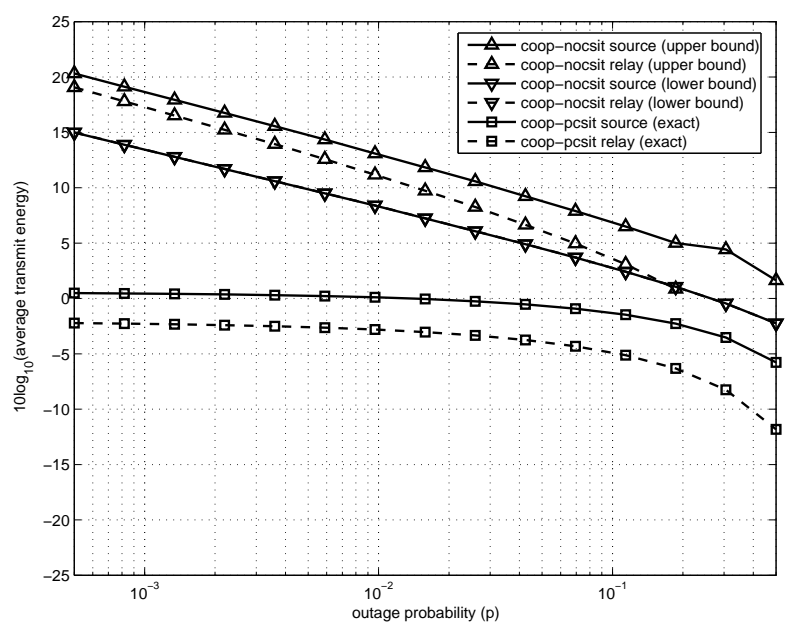

Fig. 3. Optimum energy allocation when source/relay face statistically symmetric channels to the destination. Note that the source and relay transmit with identical energy when using the lower bound (9).

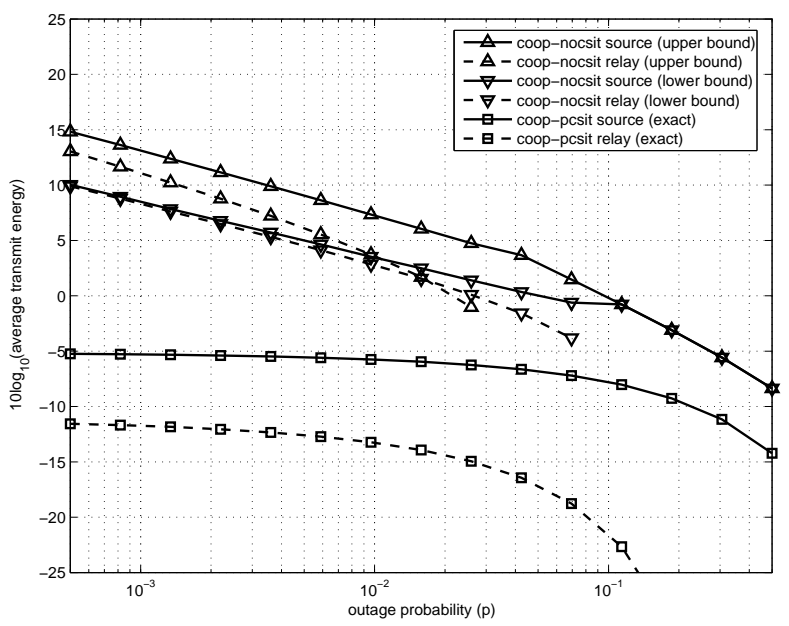

Fig. 4. Optimum energy allocation when the relay has a statistically disadvantaged channel to the destination. Note that the relay does not transmit for $p>0.07$ when using the lower bound (9).

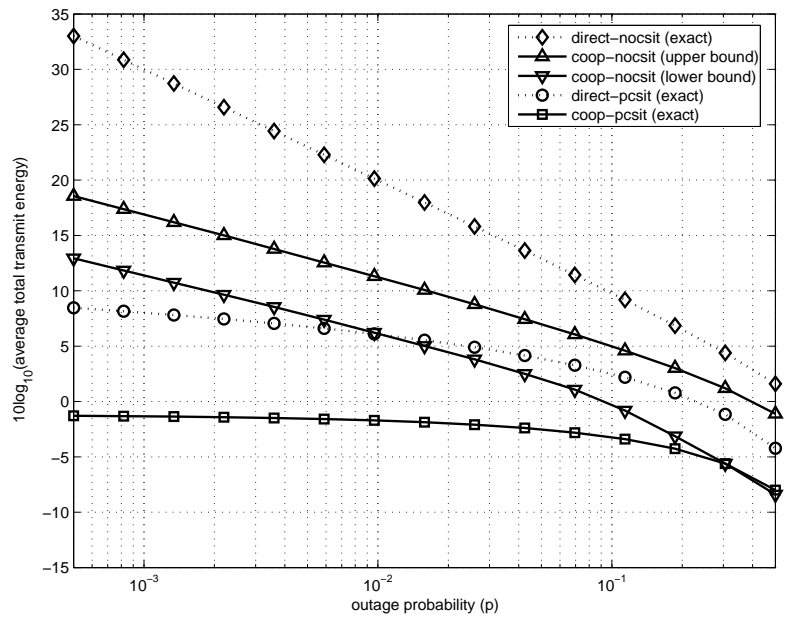

Fig. 5. Average total transmission energy of optimum cooperative and direct transmission when the relay has a statistically advantaged independent Rayleigh fading channel to the destination.

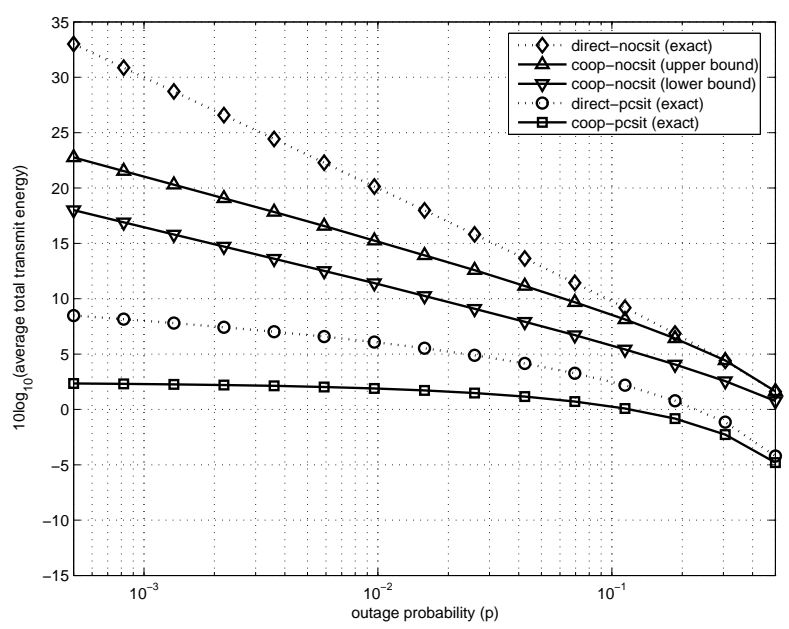

Fig. 6. Average total transmission energy of optimum cooperative and direct transmission when source/relay face statistically symmetric independent Rayleigh fading channels to the destination.

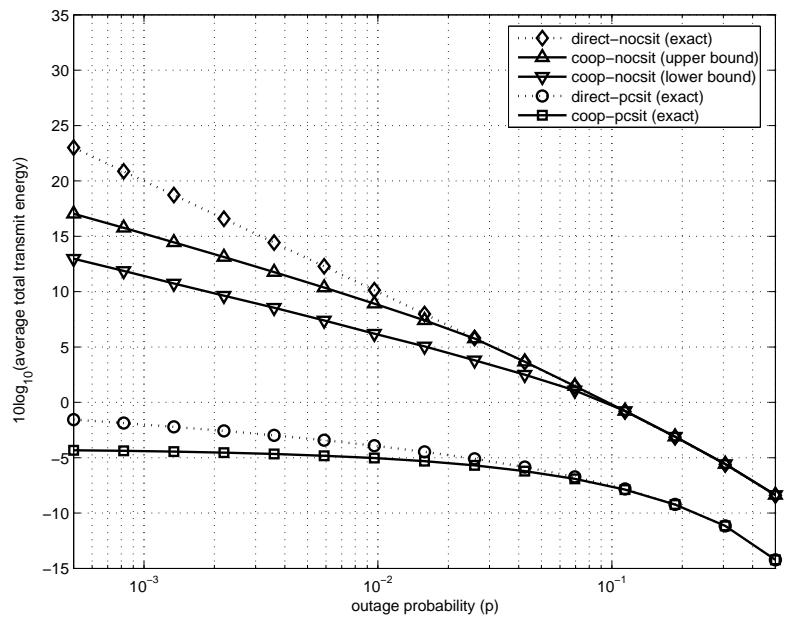

Fig. 7. Average total transmission energy of optimum cooperative and direct transmission when the relay has a statistically disadvantaged independent Rayleigh fading channel to the destination. 
with respect to the source and $p$ is sufficiently large. The lower bound energy allocations are less accurate than the upper bound due to the ideal inter-source channel assumption. When the relay has an advantaged channel, for example, the lower bound results suggest that only the relay should transmit. The lower bound results do, however, tend to agree more closely with the upper bound results when the relay channel is disadvantaged.

Figures 5, 6, and 7 show the total transmit energies needed to satisfy the outage probability target $p$ (assuming optimum energy allocation) and include results for direct transmission with and without CSIT for comparison. The results show, as expected, that cooperative transmission without CSIT achieves a fixed outage probability with less total energy than direct transmission without CSIT. Similarly, cooperative transmission with PCSIT achieves a fixed outage probability with less total energy direct transmission with PCSIT. In both cases, the energy gains tend to be large when the relay has a statistically advantaged channel to the destination and/or $p \rightarrow 0$. The potential energy gain of cooperative transmission diminishes as the relay's channel becomes less advantaged, and goes to zero at larger values of $p$ due to the fact the minimum energy strategy is for the source to use direct transmission when the relay's channel is statistically symmetric or disadvantaged and $p$ is large.

Figures 5, 6, and 7 also expose the impact of PCSIT on the overall energy efficiency the communication system shown in Figure 1. Both direct transmission and cooperative transmission are considerably more efficient when PCSIT is available. It is somewhat surprising to note, however, that direct transmission with PCSIT is more energy efficient than cooperative transmission without CSIT in almost all of the cases considered. In fact, when the relay has a statistically symmetric or disadvantaged channel to the destination, the energy required for direct transmission with PCSIT is less than even the lower bound results for cooperative transmission without CSIT for all $p$. In the case when the relay has an a statistically advantaged channel, the energy required for direct transmission with PCSIT is less than the upper bound results for cooperative transmission without CSIT for all $p$. These results demonstrate that a feedback channel providing PCSIT to a source may offer more benefit, at least in terms of transmission energy efficiency in fading channels, than cooperation without CSIT.

\section{CONCLUSIONS}

This paper examines the impact of partial channel state information (PCSIT) on optimum energy allocation and energy efficiency of a wireless communication system with two delay-constrained cooperating sources and one destination using the amplify-and-forward protocol. The sources are each required to satisfy an outage probability constraint. An explicit optimum (minimum total energy) source/relay energy allocation strategy is derived for the case when the sources have PCSIT (instantaneous channel amplitudes). For the case without CSIT, outage probability bounds are derived. Numerical examples with independent Rayleigh fading channels demonstrate that PCSIT can significantly improve the energy efficiency of both cooperative and direct transmission. The results also suggest that, while cooperative transmission tends to have better energy efficiency than direct transmission, cooperative transmission without CSIT is often less energy efficient than direct transmission with PCSIT.

\section{REFERENCES}

[1] A. Sendonaris, E. Erkip, and B. Aazhang, "Increasing uplink capacity via user cooperation diversity," in Proc. 1998 IEEE International Symp. on Information Theory, p. 156, August 16211998.

[2] A. Sendonaris, E. Erkip, and B. Aazhang, "User cooperation diversity - part i: System description," IEEE Transactions on Communications, vol. 51, pp. 1927-1938, Nov. 2003.

[3] A. Sendonaris, E. Erkip, and B. Aazhang, "User cooperation diversity - part ii: Implementation aspects and performance analysis," IEEE Transactions on Communications, vol. 51, pp. 1939-1948, Nov. 2003.

[4] J. N. Laneman, D. N. C. Tse, and G. W. Wornell, "Cooperative diversity in wireless networks: Efficient protocols and outage behavior," IEEE Transactions on Information Theory, vol. 50, no. 12 , pp. 3062-3080, 2004.

[5] A. Stefanov and E. Erkip, "Cooperative space-time coding for wireless networks," IEEE Transactions on Communications, vol. 53, no. 11, pp. 1804-1809, 2005.

[6] T. E. Hunter, S. Sanayei, and A. Nosratinia, "Outage analysis of coded cooperation," IEEE Transactions on Information Theory, vol. 52, no. 2, pp. 375-391, 2006.

[7] J. N. Laneman and G. W. Wornell, "Distributed space-timecoded protocols for exploiting cooperative diversity in wireless networks," IEEE Transactions on Information Theory, vol. 49 no. 10, pp. 2415-2425, 2003.

[8] A. Host-Madsen and J. Zhang, "Capacity bounds and power allocation for wireless relay channels," IEEE Transactions on Information Theory, vol. 51, no. 6, pp. 2020-2040, 2005.

[9] D. Gunduz and E. Erkip, "Outage minimization by opportunistic cooperation," in 2005 International Conference on Wireless Networks, Communications and Mobile Computing, vol. 2, pp. 1436-1442, 2005.

[10] M. Chen, S. Serbetli, and A. Yener, "Distributed power allocation for parallel relay networks," in IEEE Global Telecommunications Conference, vol. 3, pp. 1177-1181, 2005.

[11] J. Adeane, M. R. D. Rodrigues, and I. J. Wassell, "Centralised and distributed power allocation algorithms in cooperative networks," in 2005 IEEE 6th Workshop on Signal Processing Advances in Wireless Communications, pp. 333-337, 2005.

[12] Z. Han, T. Himsoon, W. P. Siriwongpairat, and K. J. R. Liu, "Energy-efficient cooperative transmission over multiuser ofdm networks: who helps whom and how to cooperate," in 2005 IEEE Wireless Communications and Networking Conference, vol. 2, pp. 1030-1035 Vol. 2, 2005.

[13] D. R. Brown III, "Resource allocation for cooperative transmission in wireless networks with orthogonal users," in Conference Record of the Thirty-Eighth Asilomar Conference on Signals, Systems and Computers, vol. 2, pp. 1473-1477 Vol.2, 2004.

[14] D. R. Brown III, "Resource allocation for fixed outage probability in orthogonal cooperative transmission systems," in WICAT Workshop on Cooperative Commumications, October 212005.

[15] N. Ahmed, M. A. Khojastepour, and B. Aazhang, "Outage minimization and optimal power control for the fading relay channel," in IEEE Information Theory Workshop, pp. 458-462, 2004 .

[16] J. N. Laneman, G. W. Wornell, and D. N. C. Tse, "An efficient protocol for realizing cooperative diversity in wireless networks," in Proceedings of the IEEE International Symposium on Information Theory (ISIT), p. 294, June 24-29 2001. 